\title{
Would Adding Low Doses of Lithium Salts and/or Prebiotic Fibre Interventions to an Effective Exercise Protocol Further Enhance Retention of Cognitive Integrity? Potential for Preventing Loss of Cognition with Aging Using Combinations of Low Cost Regimens
}

\author{
David A. Hart ${ }^{1,2,3,4}$ \\ ${ }^{1}$ Department of Surgery and McCaig Institute for Bone \& Joint Health, University of Calgary, Calgary, Canada; \\ ${ }^{2}$ Faculty of Kinesiology, University of Calgary, Calgary, Canada; ${ }^{3}$ Centre for Hip Health \& Mobility, University of \\ British Columbia, Vancouver, Canada; ${ }^{4}$ Bone \& Joint Health Strategic Clinical Network, Alberta Health Services, \\ Edmonton, Canada
}

Correspondence to: David A. Hart, hartd@ucalgary.ca

Keywords: Cognition, Dementia, Exercise, Lithium Salts, Sex/Gender, Obesity, Prebiotics

Received: December 7, $2017 \quad$ Accepted: January 7, $2018 \quad$ Published: January 10, 2018

Copyright $\odot 2018$ by author and Scientific Research Publishing Inc.

This work is licensed under the Creative Commons Attribution International License (CC BY 4.0).

http://creativecommons.org/licenses/by/4.0/

\section{(c) (1) Open Access}

\section{ABSTRACT}

It is clear that loss of cognition is becoming epidemic in our aging society. Onset of dementia and diseases such as Alzheimer's are very prevalent and the prognosis is not optimistic that numbers will decrease in the coming decades. Thus, this epidemic is impacting the quality of life of a large number of people, primarily females, as well as the health care systems of many countries. Of relevance is the fact that large clinical trials of candidate drugs to treat these conditions have not been overwhelming successes, indicating that we may need to take new directions or focus on prevention. One conservative approach in this regard has been the use of exercise protocols to both retain cognition and inhibit progression of loss. With the optimization of exercise protocols, it may be time to step back and ask "how can these successes be augmented to further inhibit risk and stabilize loss early in the development of these conditions?" An example of how this could be approached is via supplementation with low doses of minerals such as lithium salts, or supplementation of the diet with prebiotics in patients with obesity and metabolic syndrome. Regarding the former, recent epidemiological studies have indicated that the content of $\mathrm{Li}$ in the drinking water is associated with lower incidences of cognitive diseases/conditions. While not definitive, such clues may warrant performing controlled studies using low doses of lithium salts plus exer- 


\begin{abstract}
cise to further optimize impact on retention of cognition in those at risk, or those with early disease. Similarly, patients with obesity are at higher risk to develop dementia, and prebiotics can correct some of the metabolic derangements associated with the microbiome in such patients to impact risk. Thus, multiple low cost interventions plus exercise could further enhance retention of cognitive integrity in specific populations.
\end{abstract}

\title{
1. INTRODUCTION
}

Conditions and diseases associated with loss of cognition are rampant in the aging population [1,2], with predictions that this situation will get worse before it gets better. Interestingly, such conditions do "run in families", implying a genetic component, and they mainly affect females [ $>70 \%]$ more often than males [3-5]. However, it is unlikely that genetics, or even epigenetics could account for the tremendous increases in the incidence of dementia and loss of cognition. Some authors attribute this "epidemic" to longevity and the length of post-reproductive life, rather than "aging" per se [6]. Thus, the increases may also be related to the successful aging of the population resulting in an apparent increase due to the increased longevity in developed countries, the large increases in the population of earth resulting in larger numbers becoming more evident and now attributed to a disease process rather than a few individuals who were undiagnosed decades ago, or dramatic changes in lifestyle with many becoming more sedentary with food security [and increased risk for obesity]. These concepts regarding post-reproductive longevity thus implicate sex hormones (or the lack thereof) and potential neuroendocrine molecules in the observed sex differences $[7,8]$.

While finding pharmaceutical interventions for disease progression in this area has been the subject of intense investigation, and more information regarding details of the diseases is becoming more evident from research, the success of pharmacologic interventions to inhibit disease progression has not yet provided clear directions for going forward $[9,10]$. In part, this may be due to disease heterogeneity [11], as some subsets may be more vascular-based in nature [12], while the basis for disease in others may reside in specific brain tissues such as the hippocampus [13]. Heterogeneity may also arise due to differences in inciting events versus events related to early and late disease, or possibly due to epigenetic alterations associated with a chronic disease. This heterogeneity has implications for development of subset-specific pharmaceutical interventions [14], and may explain the lack of success with drug interventions thus far (multiple disease processes with a common endpoint?).

Furthermore, some dementia conditions also appear to have inflammatory elements [15], an aspect relevant to some of the discussion below. However, whether such inflammation is more relevant to progression than to initiation of the conditions remains to be clarified [16]. Relevant to this point are the reported links between obesity and cognitive decline/onset of dementia and risk for dementia [17-19]. In addition, it is also evident that obesity and sex (i.e. primarily females; [20]) interact to contribute to risk for dementia. Thus, women are affected by obesity more than men, and obesity increases risk of developing dementia in females more than men [21]. The relevance of obesity to dementia development may reside in the low grade inflammation/metabolic syndrome that many obese people exhibit, with excess expression of adipokines that can impact the brain [22,23]. Diet-induced obesity in rats is also reported to impact the brain in rats [24], as well as alter serum adipokine levels [25, 26]. Thus, inflammation, as well as other comorbidities, may provide a unique risk for this subset of individuals with obesity to develop dementia.

Based on the limited success of pharma-based approaches, perhaps the research focus should emphasize other directions, and building on the success of exercise programs, look to other cost-effective approaches to augment that success to impact cognitive and dementia-related diseases.

\section{EXERCISE AND COGNITION CONDITIONS}

The use of exercise to inhibit loss of cognition is been beneficial in multiple studies [3, 27-29]. Not 
only is exercise effective as evidenced by improvement in cognition test scores, but also in the activity of specific areas of the brain as assessed by functional MRI [29]. Recent studies have been attempting to optimize the exercise protocols, as well as determining whether subsets of patients may benefit more than others from exercise [3]. In this regard, it is apparent that sex-dependent variation exists in the effectiveness of exercise, with females influenced somewhat differently than males [3, 29]. Interestingly, sex differences in exercise protocol responsiveness have been noted in both older humans [30] and in older rodent models [31].

The mechanism(s) by which exercise could influence retention of cognitive integrity may be biomechanical and/or biological. Biomechanically, exercise can lead to increased blood flow to the brain and potentially, enhance the integrity and functioning of endothelial cells lining such blood vessels. Interestingly, lower shear forces appear to allow inflammatory processes to occur, while higher shear forces possibly related to aerobic exercise inhibit such processes [32]. Biologically, exercise could also induce changes in the brain, specifically in the hippocampus [33]. One possible mechanism to accomplish these changes is to enhance the integrity of the paracrine relationship between tissue-specific endothelial cells and their brain tissue counterparts. As part of any paracrine relationship is likely the exchange of metabolites, cytokines and other mediators, and growth regulators, the interactions could very likely be intrinsically linked biologically. Certainly, endothelial subsets do exist and can be responsive to a variety of signals. As exercise and mobility are central to human functioning, effective exercise programs would also impact the integrity of skeletal muscles, the whole cardiovascular system, the respiratory system, and the metabolic system in a systems biology manner.

At the present time, it is not known whether exercise programs should be optimized for the individual, or whether a "minimal" level of aerobic exercise is sufficient for most people? Based on ideas regarding exercise for other conditions [34], some "precision" in an individualized program may be important to consider. However, a very relevant question to be posed is: "Is there potential to build on exercise as a documented successful and low cost intervention to further advance effectiveness to inhibit initiation of cognitive decline and its progression by adding additional interventions?"

\section{LITHIUM SALTS AND DEMENTIA}

A number of recent epidemiological studies have provided indications that people living in areas with higher levels of lithium salts in their drinking water have lower incidence of dementia and cognition diseases [35-39]. While not an entirely new concept, the recent studies again raise awareness of the possibility that supplementing water or food with low dose lithium salts would be beneficial in this regard [35-39]. Li levels in most water sources are in the lower microgram range. Of relevance to this discussion, from those recent epidemiological reports it was not possible to ascertain physical activity levels for the de-identified individuals, nor were any sex-specific effects reported. Thus, these studies provided some level of evidence for the relationship, but details were not evident and subpopulations of patients not identified. Furthermore, the findings evaluated the association between dementia diagnosis and endogenous Li levels, and therefore, it is not known if such endogenous levels are suboptimal or optimal for impact on dementia risk.

The use of Li salts in the recent past has been focused on effectiveness in bipolar disease ever since the first report by Cade in 1949 [40]. In that specific application, the effective doses used are near toxic (0.7 $1.0 \mathrm{mmole} / \mathrm{L}$ ) and some side-effects can be experienced [41]. Li has not been identified as a natural cofactor in the functioning of enzymes and proteins, but it can influence a number of cellular and biological processes [42-44]. In addition, exactly what $\mathrm{Li}$ is targeting to be effective in bipolar disease has still not been definitively defined. Relevant to this discussion is a report that indicated that patients receiving lithium therapy (i.e. higher doses for conditions such as bipolar disorders) have a reduced prevalence of both neurological and cardiovascular disorders [45].

Natural $\mathrm{Li}$ exists as two isotopes, ${ }^{7} \mathrm{Li}(\sim 94 \%)$ and ${ }^{6} \mathrm{Li}(6 \%)$, which exhibit differing ionic radii $\left({ }^{7} \mathrm{Li}\right.$ more like $\mathrm{Mg}^{2+}$ and ${ }^{6} \mathrm{Li}$ more like $\mathrm{Na}^{+}$). As $\mathrm{Mg}$ and $\mathrm{Na}$ are common co-factors in enzymes, Li can thus impact a 
variety of important biologic systems including NaK ATPases, cyclic AMP metabolism, GSK-3beta function, and others [41-44]. Interestingly, in both humans and mice, somewhat lower doses of Li salts are effective in those of Black races and in mice with black coat color [41, 46]. Furthermore, in rodent models, $\mathrm{Li}$ salts were only effective in females and not males with regard to autoimmune diseases and obesity risk [46]. Relevant to the above discussion on exercise and the vascular system, Li can also impact endothelial cells [47]. The fact that $\mathrm{Li}$ is not an essential trace element, and can apparently influence a number of biological systems, makes it an ideal intervention as a potentially subtle modifier of systems at risk for dysregulation at low doses [41].

Levels of lithium carbonate salts are known to be elevated in European mineral springs, and even bottled Perrier water contains lithium salts $[41,42]$. While the levels of $\mathrm{Li}$ in these sources are logs lower than doses effective in bipolar diseases, based on the recent epidemiological studies [35-39], chronic exposure to such low doses of lithium salts could potentially have an influence on risk for cognitive diseases via a role as a subtle modifier of systems relevant to cognition integrity. In contrast to bipolar disease which has acute cycles of mood behavior, loss of cognition due to dementia and Alzheimer's disease is of slow onset, likely taking decades so the process could be receptive to the influences of low levels of Li. Perhaps the most effective doses when combined with exercise will be higher than those in water sources which demonstrated a potential effect on dementia [35-39], but still much lower than doses effective in bipolar diseases, conclusions that can only be derived from well controlled trials.

\section{THE PATH FORWARD-LITHIUM SALTS + EXERCISE}

It is clear that exercise is an effective intervention regarding retention of cognition. However, how could we build on that success to further enhance success, or broaden its impact? Based on the above discussion, and noting some abilities of both Li salts and exercise to target similar biological processes, one approach would be to conduct clinical trials of low dose lithium salt supplementation alone and in the context of exercise. Such trials would have to be fairly low doses since as discussed above, one would need to avoid side-effects and avoid the "more is better" mentality (avoiding the disasters of the past such as when $\mathrm{Li}$ was substituted for $\mathrm{Na}$ in diets), particularly since the trials may have to be lengthy to observe a sustained effect. In addition, trials involving Li salts may be difficult to fund from industry as Li is a natural compound and thus, cannot be patented per se. However, as higher doses of Li clearly impact brain functioning, similar to exercise protocols, one could use existing cognitive scoring systems to document impact on cognition, and fMRI tools to further assess where low dose Li salt effects were occurring in the brain. From a cost-effectiveness perspective, success in this regard could not only be focused on those with early cognitive decline, but also be employed as a potential preventative strategy. For those interested mainly in the translational impact of Li salts on cognitive diseases, natural $\mathrm{Li}$ being a combination of ${ }^{7} \mathrm{Li}$ and ${ }^{6} \mathrm{Li}$ would be sufficient to demonstrate effectiveness. However, for those more interested in mechanisms of action, purified ${ }^{7} \mathrm{Li}$ and ${ }^{6} \mathrm{Li}$ preparations could be used to elaborate more detail in this regard. In fact, the author has used such purified preparations in the past for rodent studies [46]. If Li supplementation alone leads to the identification of "responders and non-responders", then the findings could lead to new precision medicine tools to better understand disease processes in subset of patients/potential patients. If Li supplementation + exercise effectiveness is additive/synergistic, then patients win, but if the results show no additivity or even antagonism, then new insights into the mechanism(s) of action of exercise will be possible. Either set of outcomes will provide new potential abilities to also develop precision medicine approaches for patients (and potentially, pre-patients).

\section{ADDITIONAL INTERVENTIONS FOR THOSE WITH OBESITY}

Those individuals with obesity and enhanced risk for cognitive compromise and dementia may constitute a unique subset of people at risk due to the low grade inflammatory state associated with metabolic syndrome as discussed above (see Introduction). Thus, this population which has that risk profile + a potential inability to exercise effectively, may benefit from another low cost dietary intervention which could 
influence the metabolic syndrome and allow for more effective exercise. Such an intervention is the daily use of a prebiotic containing oligofructose and inulin, an intervention which has been shown to be effective in altering body composition and metabolic syndrome elements in both adults [48, 49] and children/adolescents $[50,51]$. The daily dose of prebiotic used ranges from 8 - 16 grams/day, depending on the study, as does the ratio of oligofructose to inulin [48-51]. As humans do not metabolize these prebiotics, they are apparently metabolized by gut bacteria that are part of the microbiome [52-54]. As the microbiome of people with obesity are different from those without obesity, the metabolism of the prebiotics leads to a re-establishment of some aspects of the microbiome (with accompanying decline in gut leakiness contributing to lipopolysaccharide uptake which subsequently contributes to the inflammation of the metabolic syndrome), as well as the generation of low molecular weight fatty acids that can be absorbed into the host [52-54]. Such molecules have the potential to interact with multiple host systems, including the brain to impact function, as well as re-establish metabolic regulation. Furthermore, some gut microorganisms can make neurotransmitters which can be absorbed and impact the brain via that mechanism as well [55].

Given the potential impact of a prebiotic intervention on metabolic syndrome, and enhanced body composition, it is very likely that such an intervention could reduce the risk of dementia in this population, but this would likely require long term ingestion of the prebiotic as cognitive decline/dementia may take decades to be manifest. However, once onset is diagnosed, a prebiotic intervention may still impact the rate of loss. In this regard, it is quite feasible to envision a prebiotic intervention also allowing for a more effective exercise regimen to be entertained and an evaluation of any additive or synergistic influences on cognition loss and progression of dementia. Interestingly, exercise has been reported to also influence the gut microbiota in mice [56], so the prebiotic intervention and exercise may also interact at the level of the gut microbiome as well as in the context of cognition.

Whether a three-pronged approach, containing prebiotics, exercise and lithium salts, would further benefit obese patients (or at least a subset of them) remains to be determined. In rodents, administration of lithium salts both prevented autoimmune disease progression in a murine model (but only in females), as well as weight gain and fat deposition [46]. However, human patients taking high doses of lithium salts for bipolar disease often gain weight [46]. Therefore, whether low doses of lithium salts would also be effective in conjunction with prebiotics and exercise in lessening risk for dementia remains to be defined.

\section{SUMMARY}

It is clear that exercise regimens have a positive impact on retention of cognition. However, this success raises the question: can we improve on exercise alone as a low cost intervention? The answer is that there are potentially other low cost interventions such as low dose lithium salts and/or prebiotic formulations that should be investigated and evaluated. The effectiveness of such combinations of interventions may or may not be of general impact or restricted to subsets of dementia, but clearly the huge investment in pharma has not yet paid dividends with regard to effective interventions.

Thus, exercise is likely fundamental to human integrity as we have depended on mobility to survive for thousands of years. However, in recent decades, a number of dietary, activity and physiological alterations/challenges have coincided with the "epidemic" of both obesity and dementia in an aging population. In this regard, it is interesting that the incidence of dementia/cognitive decline is more prevalent in postmenopausal females (discussed above), and thus some of the above discussed intervention combinations may work more effectively in females than males. In addition, females appear to lose their cardiovascular protection after menopause and are at higher risk for cardiovascular disease [57, 58]. Exercise has also been reported to be more effective in females in relation to cognitive decline [29] and may be more effective in the endothelial dysfunction in dementia [12], and the vascular form(s) of the condition [3]. There are also sex differences in obesity (females $>$ males) with lithium responsive conditions such as bipolar diseases [59], as well as other mental illnesses [60]. Thus, a number of lines of evidence which may give rise to the speculation that combinations of the interventions discussed should focus the initial research on 
post-menopausal female populations with mild disease or at risk for the cognitive diseases as they may respond more effectively.

Therefore, there may be multiple pathways to lose cognition integrity, but some are related to basic processes that can be addressed by simple interventions that are also low cost.

\section{ACKNOWLEDGEMENTS}

The author thanks Alberta Health Services for recent financial support, the Institute for Gender and Health of the Canadian Institutes for Health, as well as the Calgary Foundation-Grace Glaum Professorship for past support for some of the studies from the author's laboratory.

\section{REFERENCES}

1. Bellou, V., et al. (2017) Systematic Evaluation of the Associations between Environmental Risk Factors and Dementia: An Umbrella Review of Systematic Reviews and Meta-Analyses. Alzheimers Dement, 13, 406-418. https://doi.org/10.1016/j.jalz.2016.07.152

2. Hickman, R.A., et al. (2016) Alzheimer Disease and Its Growing Epidemic: Risk Factors, Biomarkers, and the Urgent Need for Therapeutics. Neurologic Clinics, 34, 941-953. https://doi.org/10.1016/j.ncl.2016.06.009

3. Barha, C.K., et al. (2017) Personalizing Exercise Recommendations for Brain Health: Considerations and Future Directions. British Journal of Sports Medicine, 51, 536-639. https://doi.org/10.1136/bjsports-2016-096710

4. Pike, C.J. (2017) Sex and the Development of Alzheimer's Disease. Journal of Neuroscience Research, 95, 671-680. https://doi.org/10.1002/jnr.23827

5. Podcasy, J.L. and Epperson, C.N. (2016) Considering Sex and Gender in Alzheimer Disease and Other Dementias. Dialogues in Clinical Neuroscience, 18, 437-446.

6. Gunn-Moore, D., et al. (2017) Alzheimer's Disease in Humans and Other Animals: A Consequence of Postreproductive Life Span and Longevity Rather Than Aging. Alzheimers Dement. [Epub ahead of Print] https://doi.org/10.1016/j.jalz.2017.08.014

7. Hamson, D.K., et al. (2016) Sex Hormones and Cognition: Neuroendocrine Influences on Memory and Learning. Comprehensive Physiology, 6, 1295-1337. https://doi.org/10.1002/cphy.c150031

8. Georgakis, M.K., et al. (2016) Age at Menopause and Duration of Reproductive Period in Association with Dementia and Cognitive Function: A Systematic Review and Meta-Analysis. Psychoneuroendocrinology, 73, 224-243. https://doi.org/10.1016/j.psyneuen.2016.08.003

9. Korczyn, A.D. (2012) Why Have We Failed to Cure Alzheimer's Disease? Journal of Alzheimers Disease, 29, 275-282. https://doi.org/10.1016/j.neurobiolaging.2012.01.061

10. Hung, S.Y. and Fu, W.M. (2017) Drug Candidates in Clinical Trials for Alzheimer's Disease. Journal of Biomedical Science, 24, 47. https://doi.org/10.1186/s12929-017-0355-7

11. Hampel, H., et al. (2017) A Precision Medicine Initiative for Alzheimer's Disease: The Road Ahead to Biomarker-Guided Integrative Disease Modeling. Climacteric, 20, 107-118.

https://doi.org/10.1080/13697137.2017.1287866

12. DiMarco, L.Y., et al. (2015) Vascular Dysfunction in the Pathogenesis of Alzheimer's Disease-A Review of Endothelium-Mediated Mechanisms and Ensuing Vicious Circles. Neurobiology of Disease, 82, 593-606. https://doi.org/10.1016/j.nbd.2015.08.014

13. Eichenbaum, H. (2017) On the Integrations of Space, Time, and Memory. Neuron, 95, 1007-1018. https://doi.org/10.1016/j.neuron.2017.06.036

14. Canevelli, M., et al. (2017) Sex and Gender Differences in the Treatment of Alzheimer's Disease: A Systematic 
Review of Randomized Controlled Trials. Pharmacological Research, 115, 218-223. https://doi.org/10.1016/j.phrs.2016.11.035

15. Bolos, M., et al. (2017) Alzheimer's Disease as an Inflammatory Disease. Biomolecular Concepts, 8, 37-43. https://doi.org/10.1515/bmc-2016-0029

16. Bagyinszky, E., et al. (2017) Role of Inflammatory Molecules in Alzheimer's Disease Progression and Diagnosis. Journal of the Neurological Sciences, 376, 242-254. https://doi.org/10.1016/j.jns.2017.03.031

17. Albanese, E., et al. (2015) Overweight and Obesity in Midlife and Brain Structure and Dementia 26 Years Later: The AGES-Reykjavik Study. American Journal of Epidemiology, 181, 672-679. https://doi.org/10.1093/aje/kwu331

18. Wang, M., et al. (2016) Metabolic, Inflammatory, and Microvascular Determinants of White Matter Disease and Cognitive Decline. American Journal of Neurodegenerative Disease, 5, 171-177.

19. Albanese, E., et al. (2017) Body Mass Index in Midlife and Dementia: Systematic Review and Meta-Regression Analysis of 589,649 Men and Women Followed in Longitudinal Studies. Alzheimer's \& Dementia, 8, 165-178. https://doi.org/10.1016/j.dadm.2017.05.007

20. Moser, V.A. and Pike, C.J. (2016) Obesity and Sex Interact in the Regulation of Alzheimer's Disease. Neuroscience \& Biobehavioral Reviews, 67, 102-118. https://doi.org/10.1016/j.neubiorev.2015.08.021

21. Sample, C.H. and Davidson, T.L. (2017) Considering Sex Differences in the Cognitive Controls of Feeding. Physiology \& Behavior. https://doi.org/10.1016/j.physbeh.2017.11.023

22. Kiliaan, A.J., et al. (2014) Adipokines: A Link between Obesity and Dementia? The Lancet Neurology, 13, 913-923. https://doi.org/10.1016/S1474-4422(14)70085-7

23. Arnoldussen, J.A., et al. (2014) Obesity and Dementia: Adipokines Interact with the Brain. European Neuropsychopharmacology, 24, 1982-1999. https://doi.org/10.1016/j.euroneuro.2014.03.002

24. Zanini, P., et al. (2017) Diet-Induced Obesity Alters Memory Consolidation in Female Rats. Physiology \& Behavior, 180, 91-97. https://doi.org/10.1016/j.physbeh.2017.08.011

25. Collins, K.H., et al. (2015) Relationship between Inflammation, the Gut Microbiota, and Metabolic Osteoarthritis Development: Studies in a Rat Model. Osteoarthritis Cartilage, 23, 1989-1998.

https://doi.org/10.1016/j.joca.2015.03.014

26. Collins, K.H., et al. (2016) A High-Fat High-Sucrose Diet Rapidly Alters Muscle Integrity, Inflammation and Gut Microbiota in Male Rats. Scientific Reports, 6, Article No. 37278. https://doi.org/10.1038/srep37278

27. Van der Wardt, V., et al. (2017) Adherence Support Strategies for Exercise Interventions in People with Mild Cognitive Impairment and Dementia: A Systematic Review. Preventive Medicine Reports, 7, 38-45. https://doi.org/10.1016/j.pmedr.2017.05.007

28. Chieffi, S., et al. (2017) Neuroprotective Effects of Physical Activity: Evidence from Human and Animal Studies. Frontiers in Neurology, 8, 188.

29. Bherer, L., et al. (2013) A Review of the Effects of Physical Activity and Exercise on Cognitive and Brain Functions in Older Adults. Journal of Aging Research, 2013, Article ID: 657508. https://doi.org/10.1155/2013/657508

30. Barha, C.K., et al. (2017) Sex Differences in Exercise Efficacy to Improve Cognition: A Systematic Review and Meta-Analysis of Randomized Controlled Trials in Older Humans. Frontiers in Neuroendocrinology, 46, 71-85. https://doi.org/10.1016/j.yfrne.2017.04.002

31. Barha, C.K., et al. (2017) Sex Differences in Aerobic Exercise Efficacy to Improve Cognition: A Systematic Review and Meta-Analysis of Studies in Older Rodents. Frontiers in Neuroendocrinology, 46, 86-105. https://doi.org/10.1016/j.yfrne.2017.06.001

32. Bryan, M.T., et al. (2014) Mechanoresponsive Networks Controlling Vascular Inflammation. Arteriosclerosis, 
Thrombosis, and Vascular Biology, 34, 2199-2205. https://doi.org/10.1161/ATVBAHA.114.303424

33. Vivar, C. and van Praag, H. (2017) Running Changes the Brain: The Long and the Short of It. Physiology (Bethesda), 32, 410-424. https://doi.org/10.1152/physiol.00017.2017

34. Hart, D.A. and Scott, A. (2012) Getting the Dose Right When Prescribing Exercise for Connective Tissue Conditions: The Yin and the Yang of Tissue Homeostasis. British Journal of Sports Medicine, 46, 696-698. https://doi.org/10.1136/bjsports-2011-090083

35. Kessing, L.V., et al. (2008) Lithium Treatment and Risk of Dementia. Arch Gen Psychiatry, 65, 1331-1335. https://doi.org/10.1001/archpsyc.65.11.1331

36. Kessing, L.V., et al. (2010) Does Lithium Protect against Dementia? Bipolar Disorders, 12, 87-94. https://doi.org/10.1111/j.1399-5618.2009.00788.x

37. Mauer, S., et al. (2014) Standard and Trace-Dose Lithium: A Systematic Review of Dementia Prevention and Other Behavioral Benefits. Australian \& New Zealand Journal of Psychiatry, 48, 809-818. https://doi.org/10.1177/0004867414536932

38. Matsunga, S., et al. (2015) Lithium as a Treatment for Alzheimer's Disease: A Systematic Review and Meta-Analysis. Journal of Alzheimer's Disease, 48, 403-410. https://doi.org/10.3233/JAD-150437

39. Kessing, L.V., et al. (2017) Association of Lithium in the Drinking Water with the Incidence of Dementia. JAMA Psychiatry, 74, 1005-1010. https://doi.org/10.1001/jamapsychiatry.2017.2362

40. Cade, J.F. (1949) Lithium Salts in the Treatment of Psychotic Excitement. The Medical Journal of Australia, 2, 349-352.

41. Hart, D.A. (1988) Immunopharmacologic Aspects of Lithium: One Aspect of a General Role as a Modulator of Homeostasis. In: Lithium: Inorganic Pharmacology and Psychiatric Use, IRL Press, Oxford, 99-102.

42. Hart, D.A. (1991) Lithium, Lymphocytes and Labyrinths: Insights into Biological Regulation and Diversity. In: Birch, N.J., Ed., The Pharmacology of Inorganic Lithium, Academic Press, New York, 289-315. https://doi.org/10.1016/B978-0-12-099300-0.50020-7

43. Hart, D.A. (1991) Lithium, Lymphocyte Stimulation and the Neuroimmune Interface. In: Galliccho, V., Ed., Lithium and the Blood, Karger, Basel, 68-78.

44. Hart, D.A., et al. (1999) Genes and Gender in Lithium Responsiveness: Parallels between Mouse and Man. In: Lucas, K.C., Becker, R.W. and Galliccho, V., Eds., Lithium-50 Years. Recent Advances in Biology and Medicine, Weider Publishing, Cheshire, 161-174.

45. Prosser, J.M. and Fieve, R.R. (2016) Patients Receiving Lithium Therapy Have a Reduced Prevalence of Neurological and Cardiovascular Disorders. Progress in Neuro-Psychopharmacology \& Biological Psychiatry, 71, 39-44. https://doi.org/10.1016/j.pnpbp.2016.06.006

46. Hart, D.A. (2016) Sex-Specific Effects of LiCl Treatment on Preservation of Renal Function and Extended Lifespan in Murine Models of SLE: Perspectives on Insights into the Potential Basis for Survivorship in NZB/NZW Female Mice. Biology of Sex Differences, 7, 31.

47. Bosche, B., et al. (2016) Low-Dose Lithium Stabilizes Human Endothelial Barrier by Decreasing MLC Phosphorylation and Universally Augments Cholinergic Vasorelaxation Capacity in a Direct Manner. Frontiers in Physiology, 7, 593.

48. Reimer, R.A., et al. (2017) Inulin-Type Fructans and Whey Protein Both Modulate Appetite by Only Fructans Alter Gut Microbiota in Adults with Overweight/Obesity: A Randomized Controlled Trial. Molecular Nutrition \& Food Research, 61. https://doi.org/10.1002/mnfr.201700484

49. Parnell, J.A., et al. (2017) Oligofructose Decreases Serum Lipopolysaccharide and Plasminogen Activator Inhibito-1 in Adults with Overweight/Obesity. Obesity (Silver Spring), 25, 510-513. 
https://doi.org/10.1002/oby.21763

50. Nicolucci, A.C. and Reimer, R.A. (2017) Prebiotics as a Modulator of Gut Microbiota in Paediatric Obesity. Pediatric Obesity, 12, 265-273. https://doi.org/10.1111/ijpo.12140

51. Nicolucci, A.C., et al. (2017) Prebiotics Reduce Body Fat and Alter Intestinal Microbiota in Children Who Are Overweight or with Obesity. Gastroenterology, 153, 711-722. https://doi.org/10.1053/j.gastro.2017.05.055

52. Puertollano, E., et al. (2014) Biological Significance of Short-Chain Fatty Acid Metabolism by the Intestinal Microbiome. Current Opinion in Clinical Nutrition \& Metabolic Care, 17, 139-144. https://doi.org/10.1097/MCO.0000000000000025

53. He, M. and Shi, B. (2017) Gut Microbiota as a Potential Target of Metabolic Syndrome: The Role of Probiotics and Prebiotics. Cell \& Bioscience, 7, 54. https://doi.org/10.1186/s13578-017-0183-1

54. O'Connor, S., et al. (2017) Prebiotics in the Management of Components of the Metabolic Syndrome. Maturitas, 104, 11-18. https://doi.org/10.1016/j.maturitas.2017.07.005

55. Dinan, T.G. and Cryan, J.F. (2017) The Microbiome-Gut-Brain Axis in Health and Disease. Gastroenterology Clinics of North America, 46, 77-89. https://doi.org/10.1016/j.gtc.2016.09.007

56. Lambert, J.E., et al. (2015) Exercise Training Modifies Gut Microbiota in Normal and Diabetic Mice. Applied Physiology, Nutrition, and Metabolism, 40, 749-752. https://doi.org/10.1139/apnm-2014-0452

57. Nasr, A. and Breckwoldt, M. (1998) Estrogen Replacement Therapy and Cardiovascular Protection: Lipid Mechanisms Are the Tip of an Iceberg. Gynecological Endocrinology, 12, 43-59. https://doi.org/10.3109/09513599809024970

58. Bani, D. (2008) Relaxin as a Natural Agent for Vascular Health. Vascular Health and Risk Management, 4, 515-524. https://doi.org/10.2147/VHRM.S2177

59. Baskaran, A., et al. (2014) Sex Differences in Rates of Obesity in Bipolar Disorder: Postulated Mechanisms. Bipolar Disorder, 16, 83-92. https://doi.org/10.1111/bdi.12141

60. Goodrich, D.E., et al. (2016) Sex Differences in Weight Loss among Veterans with Serious Mental Illness: Observational Study of a National Weight Management Program. Women's Health Issues, 26, 410-419.

https://doi.org/10.1016/j.whi.2016.05.001 